\title{
Prognostic value of receptor conversion after neoadjuvant chemotherapy in breast cancer patients: a prospective observational study
}

\author{
Xi Jin ${ }^{1, *}$, Yi-Zhou Jiang ${ }^{1, *}$, Sheng Chen ${ }^{1, *}$, Ke-Da Yu ${ }^{1}$, Zhi-Ming Shao ${ }^{1}$, Gen-Hong Di ${ }^{1}$ \\ ${ }^{1}$ Department of Breast Surgery, Fudan University Shanghai Cancer Center, Department of Oncology, Shanghai Medical \\ College, Fudan University, Shanghai 200032, China \\ *These authors have contributed equally to this work \\ Correspondence to: \\ Gen-Hong Di, e-mail: didy@medmail.com.cn \\ Yi-Zhou Jiang, e-mail: yizhoujiang@fudan.edu.cn \\ Keywords: breast cancer, neoadjuvant chemotherapy, receptor conversion, prognosis \\ Received: January 12, $2015 \quad$ Accepted: February 05, $2015 \quad$ Published: March 16, 2015
}

\section{ABSTRACT}

The hormone receptor (HR) status and human epidermal growth hormone receptor 2 (HER2) status of patients with breast cancer may change following neoadjuvant chemotherapy (NCT). This prospective observational study aimed to evaluate the prognostic impact of receptor conversion in breast cancer patients treated with NCT.Of the 423 consecutive patients who had residual disease in the breast after NCT, $55(13.0 \%)$ changed from HR (+) to HR $(-), 23(5.4 \%)$ changed from HR ( -$)$ to HR $(+), 27(6.4 \%)$ changed from HER2 (+) to HER2 (-), and $13(3.1 \%)$ changed from HER2 $(-)$ to HER2 (+). A total of $54(12.8 \%)$ changed to the triplenegative (TN) tumor phenotype. The loss of HR positivity was an independent prognostic factor for worse disease-free survival (DFS) and worse overall survival (OS) in multivariate survival analysis. Furthermore, the switch to the TN phenotype after NCT was another independent prognostic factor for worse survival for both DFS and OS. In conclusion, patients with breast cancer may experience changes in HR status, HER2 status and tumor phenotype after NCT. The loss of HR positivity and the switch to the TN phenotype after NCT were associated with a worse patient outcome.

\section{INTRODUCTION}

Neoadjuvant chemotherapy (NCT) followed by definitive surgical resection is a commonly utilized therapeutic approach for locally advanced breast cancer and is likely to improve the operability of these patients by downstaging their primary tumors [1-3]. A core needle biopsy (CNB) is commonly performed to confirm the diagnosis and determine the presence of immunohistochemical (IHC) markers, such as human epidermal growth factor receptor 2 (HER2) and hormone (estrogen and progesterone) receptor (HR), which are key factors in the decision-making process regarding adjuvant therapy as well as important prognostic indicators $[4,5]$.

Previous studies have shown that NCT can alter the status of HR [6-11] and HER2 [8, 10-13]. Patients showing a conversion from $\mathrm{HR}(+)$ to HR $(-)$ tended to benefit less from NCT compared to those with no change or the opposite conversion [from HR (-) to HR (+)] [6]. However, little information is available on the prognostic impact of receptor conversion caused by NCT. The purpose of our study was to assess the discordance rate of the HR status and the HER2 status in patients with residual tumors after NCT and to evaluate the prognostic significance of multiple changes in these statuses.

\section{RESULTS}

\section{Patient characteristics}

A total of 121 of the 544 patients (22.2\%) with primary breast cancer who received NCT in our study were considered pathologic complete response (pCR) after NCT. These complete responders were excluded from this prospective observational study due to the lack of residual tumors. The remaining 423 patients (77.8\%) with 
residual disease in the breast were included in this study (Supplemental Figure 1). Of the 423 patients, 351 patients $(83.0 \%)$ were older than 40 years and 177 patients $(41.8 \%)$ were post-menopausal. The median tumor size of the surgical specimens was three centimeters, and the average number of lymph nodes involved was four. All patients underwent one to six cycles of NCT using a regimen of PC (Paclitaxel and Carboplatin, 24.8\%), CEF (Cyclophosphamide, Epirubicin and 5-fluorouracil, 28.1\%), NE (Navelbine and Epirubicin, 39.9\%), TE (Docetaxel and Epirubicin, 6.1\%), or other agents (0.9\%). Approximately 146 patients (53.2\%) had high Ki67 expression (Table 1).

We also compared the expression of $\mathrm{Ki} 67$ in different receptor conversion groups. Significantly high Ki67 expression was observed in the groups with loss of
HR status (mean, 39.0), loss of HER2 status (mean, 36.5) and a discordant triple-negative (TN) tumor phenotype (mean, 30.2) (Figure 1).

\section{Discordance in receptor expression measurement}

Patients with HR and HER2 status conversions before and after NCT were divided into four groups: $(+)$ to $(+),(-)$ to $(-),(+)$ to $(-)$ and $(-)$ to $(+)$. The HR status of up to 78 patients $(18.4 \%)$ was converted after $\mathrm{NCT}$, and this conversion was predominantly from HR $(+)$ to $(-)$ (55 patients, $13.0 \%)$. A total of 23 patients $(5.4 \%)$ showed a change in their HR status from (-) to $(+)$. With regard to HER2 status, 40 patients $(9.5 \%)$ presented a discordant HER2 status; of these individuals, $27(6.3 \%)$ were converted from HER2 (+) to HER2 (-),

\section{Table 1: Patients and tumor characteristics}

\begin{tabular}{|c|c|c|}
\hline Variables & $n$ & $\%$ \\
\hline \multicolumn{3}{|l|}{ Age } \\
\hline$\leq 40$ years & 72 & 17.0 \\
\hline$>40$ years & 351 & 83.0 \\
\hline \multicolumn{3}{|l|}{ Menopausal Status } \\
\hline pre-menopausal & 246 & 58.2 \\
\hline post-menopausal & 177 & 41.8 \\
\hline \multicolumn{3}{|l|}{ Initial Tumor Size } \\
\hline$\leq 2 \mathrm{~cm}$ & 6 & 1.4 \\
\hline$>2$ and $\leq 5 \mathrm{~cm}$ & 114 & 27.0 \\
\hline$>5 \mathrm{~cm}$ & 198 & 46.8 \\
\hline unknown & 105 & 24.8 \\
\hline \multicolumn{3}{|l|}{ Initial Tumor Stage } \\
\hline $\mathrm{T} 1$ & 4 & 0.9 \\
\hline $\mathrm{T} 2$ & 134 & 31.7 \\
\hline $\mathrm{T} 3$ & 212 & 50.1 \\
\hline $\mathrm{T} 4$ & 73 & 17.2 \\
\hline \multicolumn{3}{|l|}{ Initial Node Status } \\
\hline negative & 107 & 25.3 \\
\hline positive & 316 & 74.7 \\
\hline \multicolumn{3}{|l|}{ Histologic Type } \\
\hline ductal & 386 & 91.3 \\
\hline lobular & 8 & 1.9 \\
\hline other & 29 & 6.9 \\
\hline \multicolumn{3}{|l|}{ Initial HR Status } \\
\hline negative & 166 & 39.2 \\
\hline positive & 257 & 60.8 \\
\hline
\end{tabular}

(Continued) 


\begin{tabular}{|c|c|c|}
\hline Variables & $n$ & $\%$ \\
\hline \multicolumn{3}{|l|}{ Initial HER2 Status } \\
\hline negative & 341 & 80.6 \\
\hline positive & 82 & 19.4 \\
\hline \multicolumn{3}{|c|}{ Pre-NCT Tumor Phenotype } \\
\hline $\operatorname{HR}(+) / \operatorname{HER} 2(-)$ & 213 & 50.3 \\
\hline $\operatorname{HR}(+) / \operatorname{HER} 2(+)$ & 44 & 10.4 \\
\hline HR $(-) /$ HER2 $(+)$ & 38 & 9.0 \\
\hline HR $(-) / \operatorname{HER} 2(-)$ & 128 & 30.3 \\
\hline \multicolumn{3}{|l|}{ NCT Regimen } \\
\hline $\mathrm{PC}$ & 105 & 24.8 \\
\hline $\mathrm{CEF}$ & 119 & 28.1 \\
\hline $\mathrm{NE}$ & 169 & 39.9 \\
\hline $\mathrm{TE}$ & 26 & 6.1 \\
\hline others & 4 & 1.0 \\
\hline \multicolumn{3}{|l|}{ NCT Cycles } \\
\hline $1-2$ & 106 & 25.1 \\
\hline $3-4$ & 272 & 64.3 \\
\hline $5-6$ & 45 & 10.6 \\
\hline \multicolumn{3}{|l|}{ Response to NCT } \\
\hline PR & 229 & 54.1 \\
\hline $\mathrm{SD} / \mathrm{PD}$ & 194 & 45.9 \\
\hline \multicolumn{3}{|c|}{ Tumor Size at Surgery } \\
\hline$\leq 2 \mathrm{~cm}$ & 152 & 35.9 \\
\hline$>2$ and $\leq 5 \mathrm{~cm}$ & 206 & 48.7 \\
\hline$>5 \mathrm{~cm}$ & 65 & 15.4 \\
\hline \multicolumn{3}{|c|}{ Number of Positive Nodes at Surgery } \\
\hline 0 & 76 & 18.0 \\
\hline $1-3$ & 127 & 30.0 \\
\hline$\geq 4$ & 220 & 52.0 \\
\hline \multicolumn{3}{|l|}{ Vascular Invasion } \\
\hline negative & 230 & 54.4 \\
\hline positive & 88 & 20.8 \\
\hline unknown & 105 & 24.8 \\
\hline \multicolumn{3}{|l|}{ Histologic Grade } \\
\hline 1 & 3 & 0.7 \\
\hline 2 & 159 & 37.6 \\
\hline 3 & 42 & 10.0 \\
\hline unknown & 219 & 51.8 \\
\hline
\end{tabular}

(Continued) 


\begin{tabular}{|c|c|c|}
\hline Variables & $n$ & $\%$ \\
\hline \multicolumn{3}{|l|}{ Ki67 at Surgery } \\
\hline $\operatorname{high}(\geq 15 \%)$ & 146 & 34.5 \\
\hline low $(<15 \%)$ & 277 & 65.5 \\
\hline \multicolumn{3}{|c|}{ HR Status at Surgery } \\
\hline negative & 198 & 46.8 \\
\hline positive & 225 & 53.2 \\
\hline \multicolumn{3}{|c|}{ HER2 Status at Surgery } \\
\hline negative & 355 & 83.9 \\
\hline positive & 68 & 16.1 \\
\hline \multicolumn{3}{|c|}{ Adjuvant Chemotherapy } \\
\hline Yes & 377 & 89.1 \\
\hline No & 46 & 10.9 \\
\hline \multicolumn{3}{|c|}{ Adjuvant Hormone Therapy } \\
\hline Yes & 230 & 54.4 \\
\hline No & 193 & 45.6 \\
\hline \multicolumn{3}{|c|}{ Adjuvant Radiotherapy } \\
\hline Yes & 281 & 66.4 \\
\hline No & 142 & 33.6 \\
\hline \multicolumn{3}{|l|}{ HR Conversion } \\
\hline$(-)$ to $(-)$ & 143 & 33.8 \\
\hline$(+)$ to $(+)$ & 202 & 47.8 \\
\hline$(+)$ to $(-)$ & 55 & 13.0 \\
\hline$(-)$ to $(+)$ & 23 & 5.4 \\
\hline \multicolumn{3}{|l|}{ HER2 Conversion } \\
\hline$(-)$ to $(-)$ & 328 & 77.5 \\
\hline$(+)$ to $(+)$ & 55 & 13.0 \\
\hline$(+)$ to $(-)$ & 27 & 6.4 \\
\hline$(-)$ to $(+)$ & 13 & 3.1 \\
\hline \multicolumn{3}{|c|}{ Tumor Phenotype Conversion } \\
\hline concordant nTN & 213 & 50.3 \\
\hline concordant TN & 110 & 26.0 \\
\hline discordant nTN & 46 & 10.9 \\
\hline discordant TN & 54 & 12.8 \\
\hline
\end{tabular}

CEF: Cyclophosphamide + Epirubicin + 5-fluorouracil; HER2: human epidermal growth factor receptor 2; HR: hormone receptor; NCT: neoadjuvant chemotherapy; NE: Navelbine + Epirubicin; nTN: non-triple-negative; PC: Paclitaxel + Cyclophosphamide; PR: partial response; SD/PD: stable disease or progression of disease; TE: Docetaxel + Epirubicin; TN: triple-negative. 

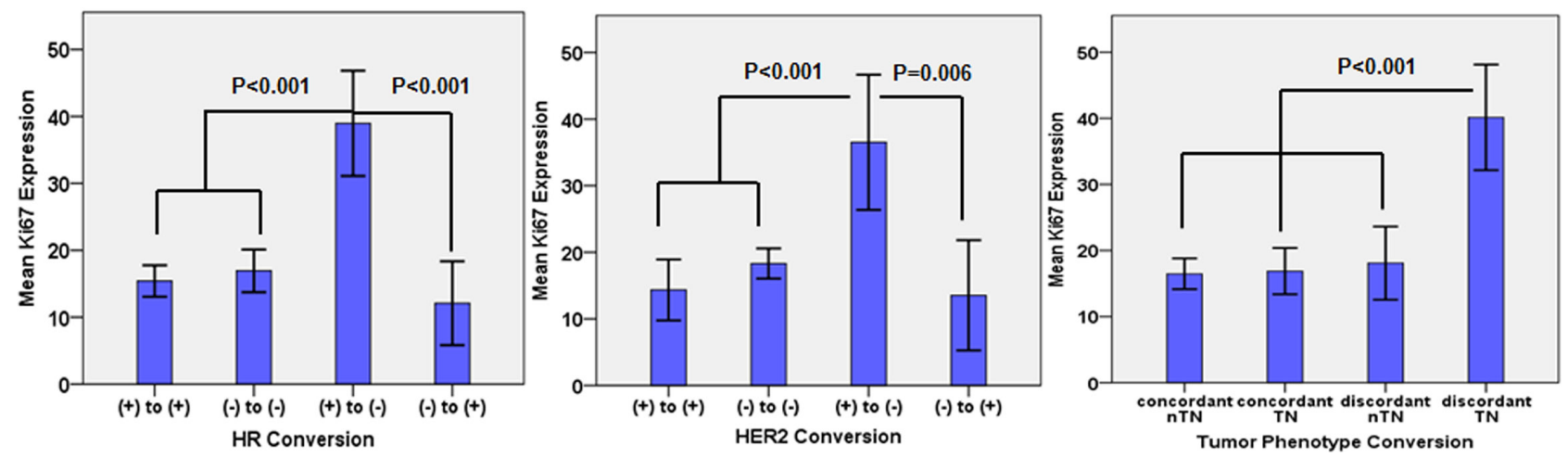

Figure 1: Comparison of Ki-67 expression in the different conversion groups. The data represent the mean \pm standard deviation of Ki67 levels. Statistical significance is indicated.

and $13(3.1 \%)$ changed from HER2 $(-)$ to HER2 $(+)$. We defined the tumor phenotypes as HR $(+) / \operatorname{HER} 2(-)$, HR $(+) / \operatorname{HER} 2(+), \operatorname{HR}(-) / \operatorname{HER} 2(+)$ and HR $(-) / \operatorname{HER} 2$ $(-)$. Discordance in tumor phenotypes was observed in 100 patients $(23.6 \%)$, and 54 patients $(12.8 \%)$ converted to TN (Table 1).

\section{Prognostic impact of HR and HER2 conversion}

Kaplan-Meier plots for disease-free survival (DFS) and overall survival (OS) according to HR conversion and HER2 conversion are shown in Figure 2. Patients showing a change in their HR status from $(+)$ to $(-)$ after NCT had significantly worse DFS than the other three groups of patients $(P<0.001)$ (Figure 2a). Similar significant differences in OS were also observed $(P<0.001)$ (Figure 2b). According to the four HER2 conversion groups, patients who remained HER2 $(-)$ after NCT had better DFS $(P<0.001)$ (Figure $2 \mathrm{c})$ and OS $(P=0.007)$ (Figure 2d).

To further evaluate the difference in patient survival based on changes in their HR and HER2 statuses after NCT, univariate and multivariate Cox regression analyses of DFS and OS were carried out. The results of univariate Cox regression analysis are shown in Supplemental Table 1. The multivariate model included all variables that were statistically significant in the univariate analysis except for interactive variables (Table 2). In the multivariate Cox regression analysis, the number of positive nodes at surgery $(P<0.001$ and $P=0.001)$, HR conversion $(P=0.001$ and $P=0.001)$ and HER2 conversion $(P<0.001$ and $P=0.003)$ were statistically significant for both DFS and OS; NCT regimens were only significant for OS $(P=0.002)$. Among the groups showing a change in HR status, patients with loss of HR positivity had significantly worse survival outcomes (Hazard ratios, HazR $=2.648, P<0.001$ for DFS; HazR $=3.460$, $P<0.001$ for OS) compared with positive patients with a concordant HR status (HazR $=1$ for DFS and OS) and patients with an HR status gain (HazR $=2.400, P=0.013$ for DFS; HazR $=3.834, P=0.008$ for OS). Loss of HER2 positivity alone was not significantly associated with DFS or OS.

To exclude the influence of hormone therapy, we next performed Kaplan-Meier plots to analyze the prognostic impact of HR conversion among 190 patients who received hormone therapy. Patients with loss of HR status after NCT still had significantly worse survival outcomes compared with patients with a concordant HR positive status $(P=0.002$ for DFS and $P=0.003$ for OS) (Supplemental Figure 2).

\section{Tumor phenotype conversion and patient outcomes}

Patients showing a conversion of the tumor phenotype were divided into four groups: concordant nonTN (nTN): the tumor phenotype was unchanged and not $\mathrm{TN}$; concordant TN: the tumor phenotype was unchanged and $\mathrm{TN}$; discordant nTN: the tumor phenotype was changed and the residual tumor was not $\mathrm{TN}$; discordant $\mathrm{TN}$ : the tumor phenotype was changed and the residual tumor was TN. We performed Kaplan-Meier analyses of the tumor phenotype conversion (Figure 3). Patients who maintained the same tumor phenotype with no changes had significantly better outcomes compared with discordant cases $(P<0.001$ for DFS and $P=0.001$ for OS) (Figure $3 a$ and $3 b)$.

Among these four groups, patients whose tumor phenotype switched to TN had the worst DFS $(\mathrm{HazR}=2.713, P<0.001)$ and OS $(\mathrm{HazR}=2.477$, $P=0.003)$ compared with patients with concordant $\mathrm{nTN}$ (HazR $=1$ for DFS and OS) and discordant nTN (HazR $=2.282, P=0.027$ for OS, not significant for DFS) in multivariate analyses. Similar results are also seen in Figure $3 \mathrm{c}$ and $3 \mathrm{~d}$. Patients with concordant TN and concordant nTN did not reach statistical significance for DFS or OS (Table 2). 


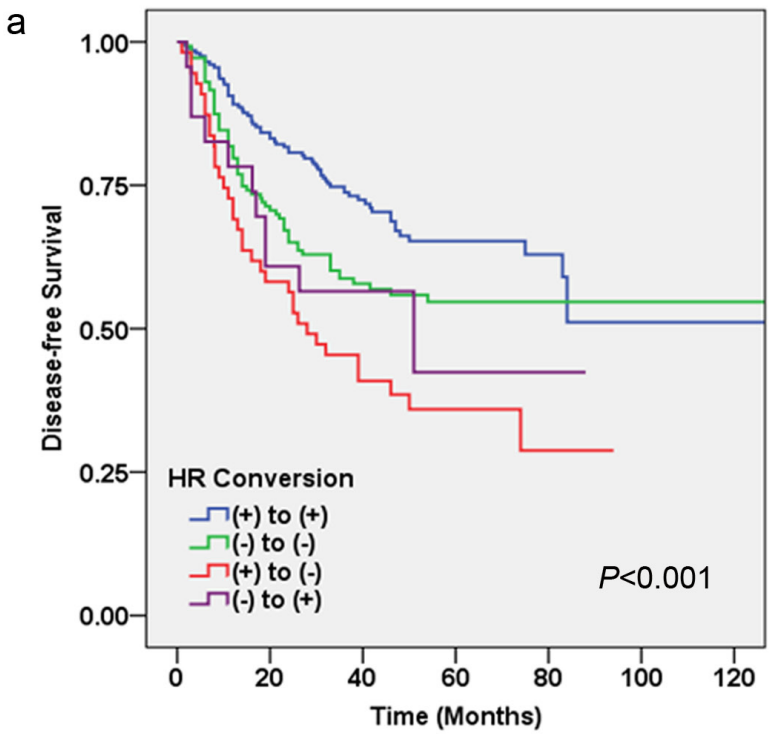

b

\begin{tabular}{cccccccc} 
& \multicolumn{7}{c}{ Numbers of patients at risk } \\
HR (+) to (+) & 202 & 170 & 105 & 55.5 & 18 & 6 & 0.5 \\
HR (-) to (-) & 143 & 102 & 61.5 & 41.5 & 12.5 & 5 & 0.5 \\
HR (+) to (-) & 55 & 32 & 18 & 14 & 3 & 0 & 0 \\
HR (-) to (+) & 23 & 14 & 7 & 3 & 1 & 0 & 0
\end{tabular}

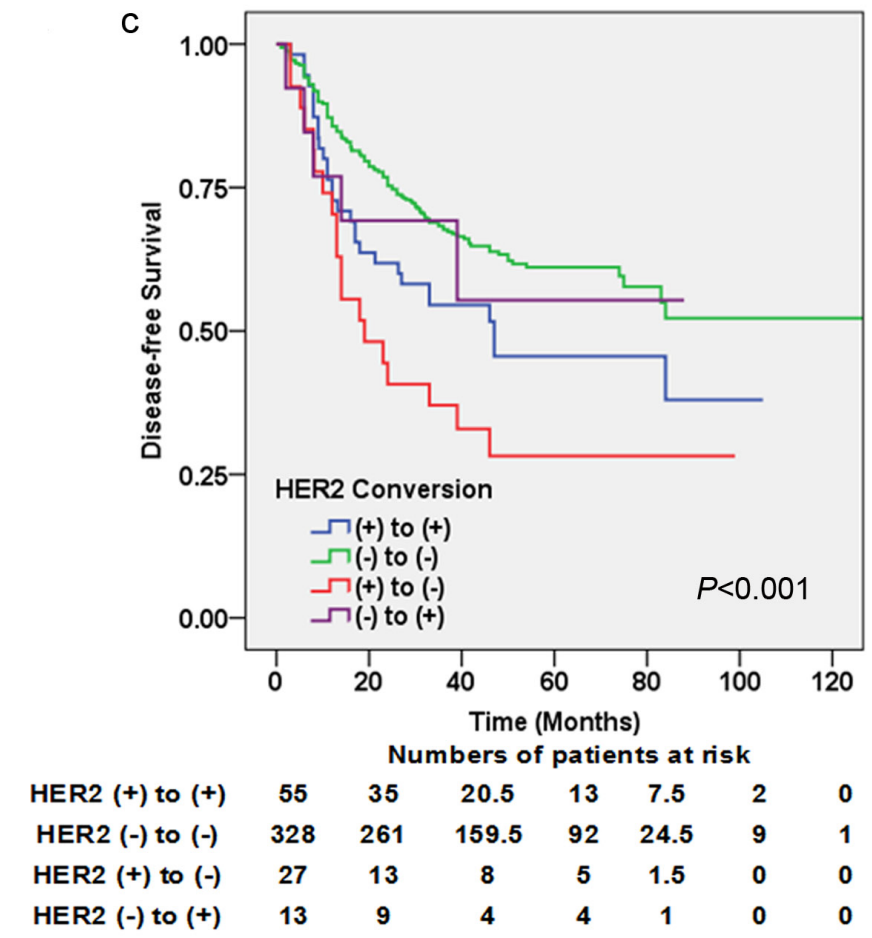

$d$
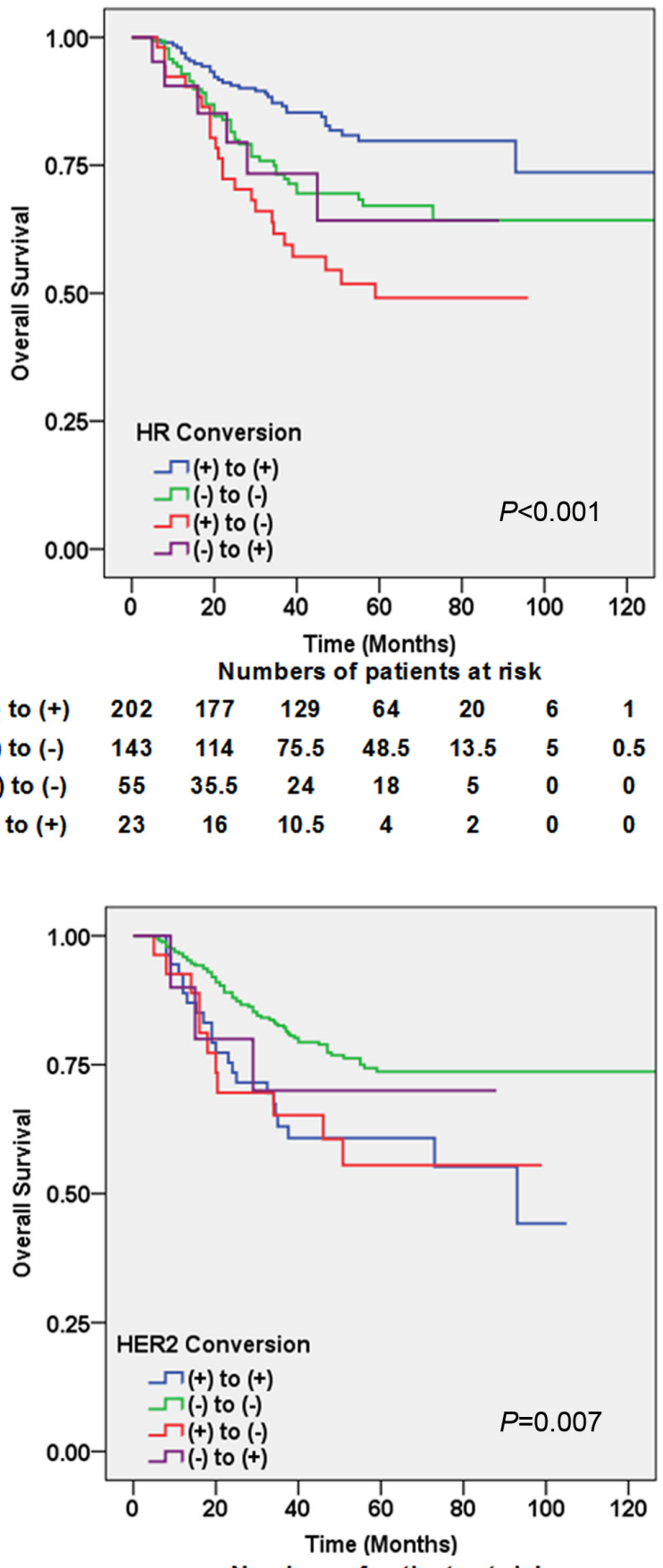

$\begin{array}{lccccccc}\text { HER2 (+) to (+) } & 55 & 41 & 26.5 & 17 & 7.5 & 2 & 0 \\ \text { HER2 (-) to (-) } & 328 & 278 & 192.5 & 103 & 29.5 & 9 & 1.5 \\ \text { HER2 (+) to (-) } & 27 & 20 & 15 & 10.5 & 2.5 & 0 & 0 \\ \text { HER2 (-) to (+) } & 13 & 8 & 5 & 4 & 1 & 0 & 0\end{array}$

Figure 2: Kaplan-Meier estimates of disease-free survival (DFS) and overall survival (OS) according to receptor conversions. a. DFS for hormone receptor (HR) conversion (log-rank test: $P<0.001$ ), b. DFS for human epidermal growth factor receptor 2 (HER2) conversion (log-rank test: $P<0.001$ ), c. OS for HR conversion (log-rank test: $P<0.001$ ), d. OS for HER2 conversion (log-rank test: $P=0.007)$. 

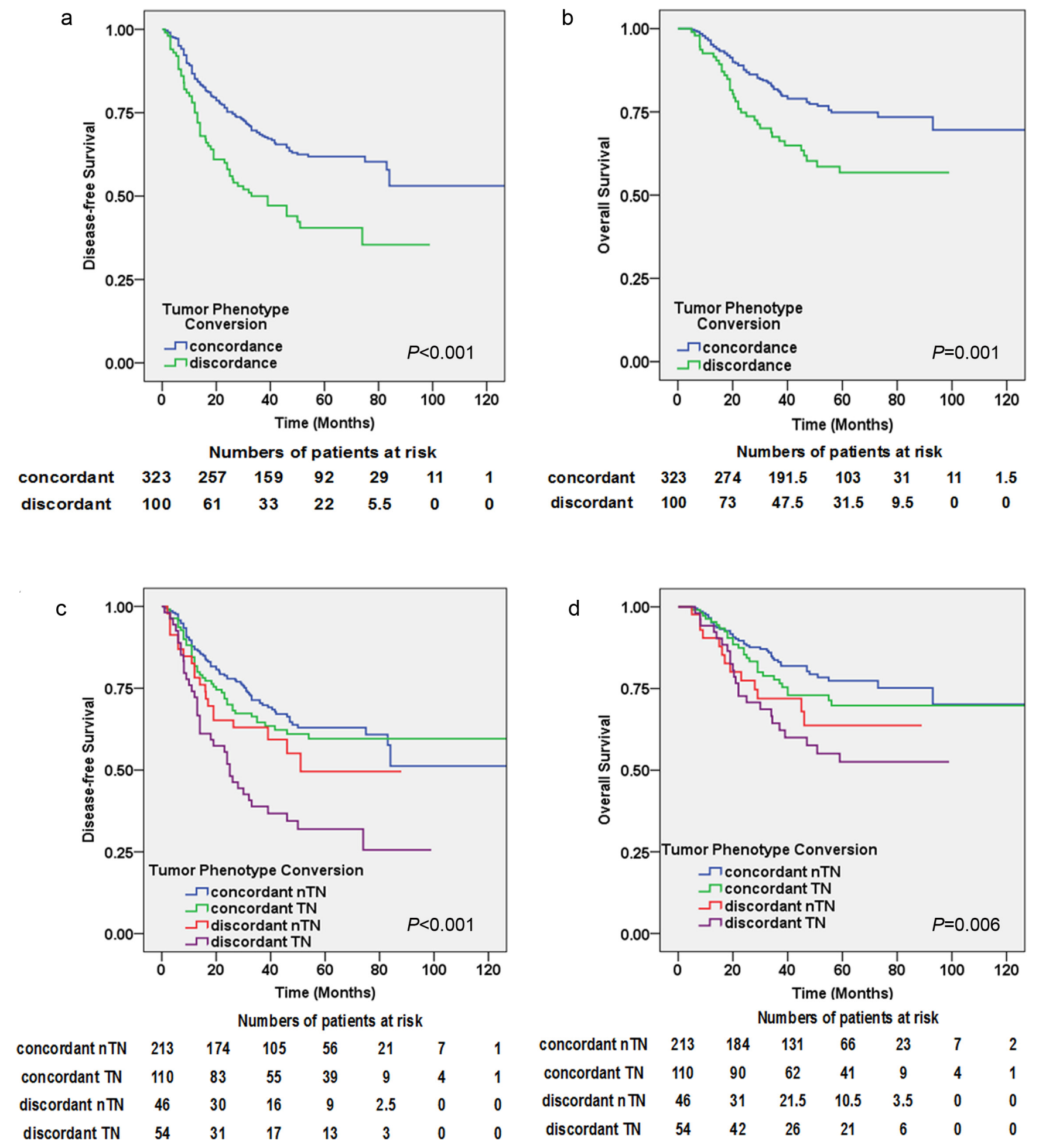

Figure 3: Kaplan-Meier estimates of DFS and OS by tumor phenotype conversion. a. DFS (log-rank test: $P<0.001$ ), b. OS (log-rank test: $P=0.001$ ). Kaplan-Meier estimates of DFS and OS for the groups with concordant triple-negative (TN) group, concordant non-TN (nTN) group, discordant TN group and discordant nTN group: c. DFS (log-rank test: $P<0.001)$, d. OS (log-rank test: $P=0.003$ ). 
Table 2: Multivariate analyses of DFS and OS of non-pCR patients with neoadjuvant chemotherapy $(n=423)$

\begin{tabular}{|c|c|c|c|c|c|c|}
\hline \multirow{3}{*}{ Variables } & \multirow{2}{*}{\multicolumn{2}{|c|}{ ALL }} & \multicolumn{4}{|c|}{ Multivariate analyses } \\
\hline & & & \multicolumn{2}{|r|}{ DFS $^{\mathbf{a}}$} & \multicolumn{2}{|c|}{$\mathbf{O S}^{\mathbf{b}}$} \\
\hline & $n$ & $\%$ & $\boldsymbol{P}$ & HazR (95\% CI) & $\boldsymbol{P}$ & HazR (95\% CI) \\
\hline HR Conversion & & & 0.001 & & 0.001 & \\
\hline$(+)$ to $(+)$ & 202 & 47.8 & & 1 & & 1 \\
\hline$(-)$ to $(-)$ & 143 & 33.8 & 0.411 & $1.268(0.720-2.235)$ & 0.596 & $1.222(0.583-2.558)$ \\
\hline$(+)$ to $(-)$ & 55 & 13.0 & $<0.001$ & $2.648(1.609-4.358)$ & $<0.001$ & $3.460(1.738-6.885)$ \\
\hline$(-)$ to $(+)$ & 23 & 5.4 & 0.013 & $2.400(1.206-4.776)$ & 0.008 & $3.834(1.416-10.377)$ \\
\hline HER2 Conversion & & & $<0.001$ & & 0.003 & \\
\hline$(+)$ to $(+)$ & 55 & 13.0 & & 1 & & 1 \\
\hline$(-)$ to $(-)$ & 328 & 77.5 & 0.033 & $0.609(0.386-0.961)$ & 0.005 & $0.434(0.243-0.776)$ \\
\hline$(+)$ to $(-)$ & 27 & 6.4 & 0.058 & $1.856(0.980-3.515)$ & 0.687 & $1.184(0.520-2.695)$ \\
\hline$(-)$ to $(+)$ & 13 & 3.1 & 0.299 & $0.561(0.189-1.671)$ & 0.248 & $0.399(0.084-1.895)$ \\
\hline $\begin{array}{l}\text { Tumor Phenotype } \\
\text { Conversion }\end{array}$ & & & $<0.001$ & & 0.001 & \\
\hline concordant nTN & 213 & 50.3 & & 1 & & 1 \\
\hline concordant TN & 110 & 26.0 & 0.545 & $0.862(0.532-1.395)$ & 0.527 & $0.825(0.454-1.498)$ \\
\hline discordant nTN & 46 & 10.9 & 0.060 & $1.691(0.978-2.922)$ & 0.027 & 2.282(1.099-4.739) \\
\hline discordant $\mathrm{TN}$ & 54 & 12.8 & $<0.001$ & $2.713(1.718-4.284)$ & 0.003 & $2.477(1.373-4.467)$ \\
\hline
\end{tabular}

CI: confidence interval; DFS: disease-free survival; HazR: hazard ratio; HER2: human epidermal growth factor receptor 2; HR: hormone receptor; nTN: non-triple-negative; OS: overall survival; TN: triple-negative.

${ }^{a}$ The DFS multivariate analyses were adjusted for initial node status, NCT cycles, response to NCT, tumor size at surgery, number of positive nodes at surgery and adjuvant hormone therapy.

${ }^{b}$ The OS multivariate analyses were adjusted for initial tumor status, initial node status, NCT regimens, tumor size at surgery, number of positive nodes at surgery and adjuvant hormone therapy.

\section{DISCUSSION}

Previous studies have presented conflicting results regarding the conversion of the HR status and the HER2 status of patients with breast cancer during NCT. While several studies have suggested that the expression of these receptors is altered after NCT [6-13], others indicated that they remained stable [14-17]. Few prospective studies have focused on the prognostic value of a discordant status. In our prospective observational study, we demonstrated that patients showing a conversion from HR $(+)$ to HR (-) in their residual tumors after NCT had a worst outcome (with or without hormonal therapy) compared with other types of HR conversions. The conversion of HER2 status alone did not have a significant impact on the prognosis. A switch to TN breast cancer was associated with a worse outcome compared to that of patients with concordant TN and discordant nTN (only significant for OS).
The mechanism of the conversion of HR and HER2 status after NCT is complex. It is important to note that intratumoral heterogeneity can result in the presence of several different clones with different phenotypes within individual tumors $[18,19]$. Within the same tumor, some clones are HR $(+)$, while others are HR $(-)$. Likewise, HER2 $(+)$ cells are also not distributed evenly within individual tumors. The sensitivity to chemotherapy differs between different clones. Tumor cells that are HR (-) are more sensitive to chemotherapy than HR $(+)$ tumors, and HR $(+)$ cells, known as insensitive tumor cells [20-22], are left behind as part of the residual disease after NCT [8, 23]. Likewise, Thor et al [24], Quddus et al [13] and Wang et al [25] have documented that HER2 (+) tumor cells are more likely to be eliminated by chemotherapy, and patients with a high percentage of HER2-positive tumor cells showed a good pathologic response. We concluded that the differential sensitivity to NCT caused by the heterogeneity 
of clones inside individual tumors may account for the change in HR from $(-)$ to $(+)$ and the change in HER2 from $(+)$ to $(-)$ after NCT.

Another known mechanism of HR status conversion is the downregulation of the estrogenic hormone receptor caused by NCT itself. Bines et al and Rose et al described that chemotherapy can suppress ovarian function and adrenal glands [26, 27], and the decrease in the circulating levels of hormone caused by this suppression may alter the HR status of residual tumors from $(+)$ to $(-)$ after NCT [8]. This mechanism is considered to be the main cause for the switch of HR (+) to HR (-) after NCT. However, falsenegative identification of the HR status and the HER2 status in CNB due to intratumoral heterogeneity has been reported previously [28]. The conversion from $\mathrm{HR}(-)$ to HR (+) and HER2 (-) to HER2 (+) may be due to the availability of tumor material for CNB because CNB may represent only a small proportion of clones of different phenotypes. Other explanations for the conversion of receptor status include genetic mutations [29, 30], statistical errors and staining techniques [12].

Our research highlighted the prognostic value of the discordance in IHC status and tumor phenotype after NCT using the Kaplan-Meier plots and Cox regression. We assessed the expression of Ki67 in surgical specimens and observed relatively high levels of $\mathrm{Ki} 67$ expression in patients with loss of HR status and alteration to the TN phenotype after NCT. Ki67 is known to be a cellular proliferation marker [31, 32], and tumors with high expression of $\mathrm{Ki} 67$ exhibit relatively more aggressive behavior [6]. The poor outcome of patients associated with the conversion of HR status and the switch to the TN phenotype after NCT might be the result of a high proportion of proliferating cancer cells and their biological behavior.

Inevitably, our study has several limitations. First, as a prospective observational study, but not a clinical trial, the NCT regimens we used were not uniform. It is not easy to determine whether the receptor conversion is attributable to a special agent or to several ones. Furthermore, our study design did not evaluate the contribution of anti-HER2 therapy. Future studies will be needed to validate the prognostic value of receptor conversion in prospective cohorts. Finally, the molecular mechanisms underlying receptor conversions are uncertain.

In conclusion, our prospective observational study demonstrated the existence of discordance in the HR status and HER2 status after NCT and the negative prognostic impact of the loss of receptor positivity. These findings might help optimize the choice of sequential adjuvant therapy and improve patient survival. The administration of NCT might be the main reason for the change in receptor status, but the mechanism needs to be characterized. In the future, further studies are required to identify the mechanism for this switch in receptor status after NCT and to validate the prognostic impact associated with this switch.

\section{MATERIALS AND METHODS}

\section{Case selection}

This prospective observational study was initiated in 2003. We enrolled patients who were diagnosed with primary breast cancer and received NCT followed by modified radical mastectomy at Fudan University Shanghai Cancer Center (FUSCC) between January 1, 2003 and December 31, 2009. Patients who had received any type of treatment prior to NCT or who had metastatic disease prior to surgery were not eligible for this study. Cases of bilateral breast cancer, male breast cancer and inflammatory breast cancer were also excluded. For each participant, we collected pre-NCT CNB samples and post-NCT surgical specimens. Out of the 544 patients, we further excluded an additional 121 patients who were considered to have pCR after NCT. In total, 423 eligible patients with residual invasive tumors were included in this prospective observational study.

Data on the medical history, patient characteristics (including age, menopausal status, tumor size, tumor status, node status, histologic type, HR status, HER2 status, Ki67 expression at surgery, tumor phenotype, NCT regimens and cycles, response to NCT, vascular invasion, histologic grade and adjuvant therapies), local and distant extent of disease (evaluated by chest CT, bone scan, abdominal ultrasound, bilateral mammography, breast ultrasound or breast MRI), and pathological assessments of morphological and biological features were collected. CNB was performed to confirm the diagnosis of invasive breast cancer prior to NCT and to evaluate the HR and HER2 status. To minimize the influence of tumor heterogeneity, at least two core samples were obtained in each of the tumors.

All patients were followed up every three months for the first year and every 6 months until death. Follow-up was completed on December 31, 2013. The median length of follow-up was 44 months (range, 2 to 149 months).

This study was approved by the Ethics Committee of FUSCC, and each participant signed an informed consent document.

\section{Treatment}

The patients in our study received an NCT regimen consisting of NE (Navelbine and Epirubicin), CEF (Cyclophosphamide, Epirubicin and 5-fluorouracil), TE (Docetaxel and Epirubicin), PC (Paclitaxel and Carboplatin) or other agents for a median of 3 cycles (range, 1-6 cycles). The pCR was defined as complete disappearance of invasive carcinoma in the breast and regional lymph nodes. The clinical response to NCT was 
evaluated by physical and imaging examinations according to RECIST 1.1. The clinical response was regarded as a partial response $(\mathrm{PR})$ if the reduction in the greatest tumor diameter exceeded $30 \%$. Tumor reduction of less than $30 \%$ or an increase of up to $20 \%$ in the greatest diameter was regarded as stable disease (SD). An increase of more than $20 \%$ in the greatest diameter of the tumor or the appearance of new disease was regarded as disease progression (PD).

Mastectomy and axillary lymph node dissection were performed within four weeks of the completion of NCT. Additional cycles of chemotherapy, including anthracyclinebased and/or taxane-based regimens, were administered after the surgery; a total of six to eight cycles of chemotherapy were completed at the discretion of the treating physician on the basis of clinical and pathologic evaluations after surgery. Radiation therapy was offered at the discretion of the treating radiation oncologist after completion of adjuvant chemotherapy. Five-year standard endocrine therapy (tamoxifen for premenopausal patients, aromatase inhibitor for postmenopausal patients or sequential tamoxifen and aromatase inhibitor) was administered to 190 patients with HR (+) status pre- or post-NCT. Trastuzumab was recommended for HER2 $(+)$ patients in the adjuvant setting but was not included in any pre-operative treatment.

\section{Pathology}

Immunohistochemical analysis was performed in formalin-fixed, paraffin-embedded tissue sections using standard procedures for breast tumor specimens from $\mathrm{CNB}$ and surgical resections. HR status and HER2 status were evaluated before and after NCT, but the Ki67 index was only available for surgical specimens. Each specimen was examined independently by two experienced pathologists. Data regarding the expression of HR and HER2 were collected from a database at the pathological center of FUSCC. The cut-off value for ER positivity and PR positivity was set at $1 \%$ of tumor cells with positive nuclear staining. HER2 $(+)$ status was defined as $3(+)$ according to circumferential membrane-bound staining (HercepTest; Dako Cytomation) or amplification confirmed by fluorescence in situ hybridization (FISH). Ki67 expression was divided into two groups: Ki67 index $\geq 15 \%$ (high expression) and Ki67 index $<15 \%$ (low expression) [6]. The following antibodies were used for IHC: ER (M7047, clone 1D5, Dako, Produktionsvej, Glostrup, Denmark), PR (M3569, clone PgR636, Dako), HER2 (A0485, polyclonal rabbit antibody, Dako) and Ki-67 (M7240, clone MIB-1, Dako).

\section{Statistical analysis}

DFS was calculated from the date of surgery to the date of disease relapse (local, regional or distant relapse), the diagnosis of contralateral breast cancer or death from any cause. OS was calculated from the date of diagnosis to the date of death or last follow-up. Survival curves were estimated using the Kaplan-Meier method, and the log-rank test was used to test for differences between groups. HazR and their 95\% confidence intervals (CIs) were calculated using the Cox regression model. Multivariate Cox proportional hazard analyses were performed by adjusting for possible prognostic variables $(P<0.05$ in univariate analysis $)$ using a stepwise selection method. One-way ANOVA was used to evaluate the differences in variables among multiple groups. The Bonferroni test was performed when necessary. The results were considered statistically significant if the $P$ was $<0.05$. The statistical analysis was carried out using SPSS (version 20.0; SPSS Company, Chicago, IL).

\section{ACKNOWLEDGMENTS}

The authors are grateful to Jiong Wu, GuangYu Liu and Zhen-Zhou Shen for their excellent data management. This work was supported by grants from the National Natural Science Foundation of China (81372848, 81370075); the Municipal Project for Developing Emerging and Frontier Technology in Shanghai Hospitals (SHDC12010116); the Cooperation Project of Conquering Major Diseases in Shanghai Municipality Health System (2013ZYJB0302); the Innovation Team of Ministry of Education (IRT1223); and the Shanghai Key Laboratory of Breast Cancer (12DZ2260100).

\section{Authors' contributions}

JX and YZJ contributed to the conception of the study, data analysis and interpretation, and writing the manuscript. CS made tissue sections and participated in immunohistochemical analysis. YKD, DGH and SZM contributed to the collection and assembly of data. All authors read and approved the final manuscript.

\section{COMPETING INTERESTS}

The authors declare that they have no conflict of interest.

\section{Editorial note}

This paper has been accepted based in part on peerreview conducted by another journal and the authors' response and revisions as well as expedited peer-review in Oncotarget.

\section{REFERENCES}

1. Hortobagyi GN. Comprehensive management of locally advanced breast cancer. Cancer. 1990; 66:1387-1391.

2. Schwartz GF, Hortobagyi GN. Proceedings of the consensus conference on neoadjuvant chemotherapy in carcinoma of the breast, April 26-28, 2003, Philadelphia, Pennsylvania. Cancer. 2004; 100:2512-2532. 
3. Mauri D, Pavlidis N, Ioannidis JPA. Neoadjuvant Versus Adjuvant Systemic Treatment in Breast Cancer: A MetaAnalysis. J Natl Cancer Inst. 2005; 97:188-194.

4. Bartlett JM, Brookes CL, Robson T, van de Velde CJ, Billingham LJ, Campbell FM, Grant M, Hasenburg A, Hille ET, Kay C, Kieback DG, Putter H, Markopoulos C, et al. Estrogen receptor and progesterone receptor as predictive biomarkers of response to endocrine therapy: a prospectively powered pathology study in the Tamoxifen and Exemestane Adjuvant Multinational trial. J Clin Oncol. 2011; 29:1531-1538.

5. Chang J, Clark GM, Allred DC, Mohsin S, Chamness G, Elledge RM. Survival of patients with metastatic breast carcinoma: importance of prognostic markers of the primary tumor. Cancer. 2003; 97:545-553.

6. Chen S, Chen CM, Yu KD, Zhou RJ, Shao ZM. Prognostic value of a positive-to-negative change in hormone receptor status after neoadjuvant chemotherapy in patients with hormone receptor-positive breast cancer. Ann Surg Oncol. 2012; 19:3002-3011.

7. Lee SH, Chung MA, Quddus MR, Steinhoff MM, Cady B. The effect of neoadjuvant chemotherapy on estrogen and progesterone receptor expression and hormone receptor status in breast cancer. Am J Surg. 2003; 186:348-350.

8. van de Ven S, Smit VT, Dekker TJ, Nortier JW, Kroep JR. Discordances in ER, PR and HER2 receptors after neoadjuvant chemotherapy in breast cancer. Cancer Treat Rev. 2011; 37:422-430.

9. Zhang N, Moran MS, Huo Q, Haffty BG, Yang Q. The hormonal receptor status in breast cancer can be altered by neoadjuvant chemotherapy: a meta-analysis. Cancer Invest. 2011; 29:594-598.

10. Burcombe RJ, Makris A, Richman PI, Daley FM, Noble S, Pittam M, Wright D, Allen SA, Dove J, Wilson GD. Evaluation of ER, PgR, HER-2 and Ki-67 as predictors of response to neoadjuvant anthracycline chemotherapy for operable breast cancer. Br J Cancer. 2005; 92:147-155.

11. Shet T, Agrawal A, Chinoy R, Havaldar R, Parmar V, Badwe R. Changes in the tumor grade and biological markers in locally advanced breast cancer after chemotherapy - implications for a pathologist. Breast $\mathrm{J}$. 2007; 13:457-464.

12. Adams AL, Eltoum I, Krontiras H, Wang W, Chhieng DC. The effect of neoadjuvant chemotherapy on histologic grade, hormone receptor status, and HER2/neu status in breast carcinoma. Breast J. 2008; 14:141-146.

13. Quddus RM, Sung JC, Zhang C, Pasqueriello T, Eklund M, Steinhoff MM. HER-2/neu Expression in Locally Advanced Breast Carcinomas: Pre- and Post-Neoadjuvant Chemotherapy. Breast Cancer. 2005; 12:294-298.

14. Penault-Llorca F, Cayre A, Bouchet Mishellany F, Amat S, Feillel V, Le Bouedec G, Ferrière J, De Latour M, Chollet P. Induction chemotherapy for breast carcinoma: predictive markers and relation with outcome. Int J Oncol. 2003; 22:1319-1325.
15. Dowsett M, Ebbs SR, Dixon JM, Skene A, Griffith C, Boeddinghaus I, Salter J, Detre S, Hills M, Ashley S, Francis S, Walsh G, Smith IE. Biomarker changes during neoadjuvant anastrozole, tamoxifen, or the combination: influence of hormonal status and HER-2 in breast cancer-a study from the IMPACT trialists. J Clin Oncol. 2005; 23:2477-2492.

16. Arens N, Bleyl U, Hildenbrand R. HER2/neu, p53, Ki67, and hormone receptors do not change during neoadjuvant chemotherapy in breast cancer. Virchows Arch. 2005; 446:489-496.

17. Kasami M, Uematsu T, Honda M, Yabuzaki T, Sanuki J, Uchida Y, Sugimura H. Comparison of estrogen receptor, progesterone receptor and Her-2 status in breast cancer pre- and post-neoadjuvant chemotherapy. Breast. 2008; 17:523-527.

18. Bertos NR, Park M. Breast cancer - one term, many entities? J Clin Invest. 2011; 121:3789-3796.

19. Dent R, Trudeau M, Pritchard KI, Hanna WM, Kahn HK, Sawka CA, Lickley LA, Rawlinson E, Sun P, Narod SA. Triple-negative breast cancer: clinical features and patterns of recurrence. Clin Cancer Res. 2007; 13:4429-4434.

20. Precht LM, Lowe KA, Atwood M, Beatty JD. Neoadjuvant chemotherapy of breast cancer: tumor markers as predictors of pathologic response, recurrence, and survival. Breast J. 2010; 16:362-368.

21. Caudle AS, Gonzalez-Angulo AM, Hunt KK, Liu P, Pusztai L, Symmans WF, Kuerer HM, Mittendorf EA, Hortobagyi GN, Meric-Bernstam F. Predictors of tumor progression during neoadjuvant chemotherapy in breast cancer. J Clin Oncol. 2010; 28:1821-1828.

22. Tan MC, Al Mushawah F, Gao F, Aft RL, Gillanders WE, Eberlein TJ, Margenthaler JA. Predictors of complete pathological response after neoadjuvant systemic therapy for breast cancer. Am J Surg. 2009; 198:520-525.

23. Tacca O, Penault-Llorca F, Abrial C, Mouret-Reynier MA, Raoelfils I, Durando X, Achard JL, Gimbergues P, Cure H, Chollet $\mathrm{P}$. Changes in and prognostic value of hormone receptor status in a series of operable breast cancer patients treated with neoadjuvant chemotherapy. The oncologist. 2007; 12:636-643.

24. Thor AD, Berry DA, Budman DR, Muss HB, Kute T, Henderson IC, Barcos M, Cirrincione C, Edgerton S, Allred C, Norton L, Liu ET. erbB-2, p53, and efficacy of adjuvant therapy in lymph node-positive breast cancer. J Natl Cancer Inst. 1998; 90:1346-1360.

25. Wang J, Buchholz TA, Middleton LP, Allred DC, Tucker SL, Kuerer HM, Esteva FJ, Hortobagyi GN, Sahin AA. Assessment of histologic features and expression of biomarkers in predicting pathologic response to anthracycline-based neoadjuvant chemotherapy in patients with breast carcinoma. Cancer. 2002; 94:3107-3114.

26. Bines J, Oleske DM, Cobleigh MA. Ovarian function in premenopausal women treated with adjuvant chemotherapy for breast cancer. J Clin Oncol. 1996; 14:1718-1729. 
27. Rose DP, Davis TE. Effects of Adjuvant Chemohormonal Therapy on the Ovarian and Adrenal Function of Breast Cancer Patients. Cancer Res. 1980; 40:4037-4047.

28. Arnedos M, Nerurkar A, Osin P, A'Hern R, Smith IE, Dowsett $M$. Discordance between core needle biopsy (CNB) and excisional biopsy (EB) for estrogen receptor (ER), progesterone receptor (PgR) and HER2 status in early breast cancer (EBC). Ann Oncol. 2009; 20:1948-1952.

29. Buchholz TA, Stivers DN, Stec J, Ayers M, Clark E, Bolt A, Sahin AA, Symmans WF, Hess KR, Kuerer HM, Valero V, Hortobagyi GN, Pusztai L. Global gene expression changes during neoadjuvant chemotherapy for human breast cancer. Cancer J. 2002; 8:461-468.
30. Lee SC, Xu X, Lim YW, Iau P, Sukri N, Lim SE, Yap HL, Yeo WL, Tan P, Tan SH, McLeod H, Goh BC. Chemotherapy-induced tumor gene expression changes in human breast cancers. Pharmacogenetics and genomics. 2009; 19:181-192.

31. Gerdes J, Schwab U, Lemke H, Stein H. Production of a mouse monoclonal antibody reactive with a human nuclear antigen associated with cell proliferation. Int J Cancer. 1983; 31:13-20.

32. Urruticoechea A, Smith IE, Dowsett M. Proliferation marker Ki-67 in early breast cancer. J Clin Oncol. 2005; 23:7212-7220. 